\title{
Theoretical Analysis and Experimental Verification of Particle Damper-Based Energy Dissipation with Applications to Reduce Structural Vibration
}

\author{
Wangqiang Xiao, ${ }^{1}$ Lina Jin, ${ }^{2}$ and Binqiang Chen ${ }^{1}$ \\ ${ }^{1}$ Department of Mechanical and Electrical Engineering, Xiamen University, Xiamen 361005, China \\ ${ }^{2}$ Institute of Nuclear and New Energy Technology, Tsinghua University, Beijing 100084, China \\ Correspondence should be addressed to Binqiang Chen; 412639547@qq.com
}

Received 29 September 2014; Accepted 12 December 2014

Academic Editor: Changjun Zheng

Copyright (C) 2015 Wangqiang Xiao et al. This is an open access article distributed under the Creative Commons Attribution License, which permits unrestricted use, distribution, and reproduction in any medium, provided the original work is properly cited.

\begin{abstract}
Particle damping technology can greatly reduce vibration of equipment and structure through friction and inelastic collisions of particles. An energy dissipation model for particle damper has been presented based on the powder mechanics and the collision theory. The energy dissipation equations of friction and collision motion are developed for the particle damper. The rationality of energy dissipation model has been verified by the experiment and the distributions for the energy dissipation of particles versus acceleration are nonlinear. As the experiment process includes lots of factors of energy dissipation, such as the noise and the air resistance, the experimental value is about $7 \%$ more than the simulation value. The simulation model can provide an effective method for the design of particle damper. And the particle parameters for damper have been investigated. The results have shown that choosing an appropriate particle density, particle size, and particle filling rate determined based on the simulation model will provide the optimal damping effect for the practical application of particle damping technology.
\end{abstract}

\section{Introduction}

Particle damping technology can greatly reduce vibration of equipment and structure by friction and inelastic collisions of particles $[1,2]$. This technology has lots of advantages, for example, reduced impact force, simple structure, low costs, small modification of original structure, low additional mass, and good adaptability to a wide temperature range. These unique features provide for broad engineering prospects in aerospace, automobile and precision machinery, and so forth $[3,4]$.

The mechanism of energy dissipation for particle damping is involved in the mechanical behavior and particulate matter dynamics. The high damping performance in vibration reduction has led to advances through research $[5,6]$. Now, the research progress in the field is at an exploratory stage. The research methods are mostly divided into the simulation and the test [7-9]. The simulation method mainly includes the DEM (discrete element method), the regression analysis, and the optimization design [10-17]. Because the nonlinear contact model has a limit on the time step of contact parameters, DEM is inappropriate for large numerical computation when particle number is more than $10^{7}$ [10]. In fact, the number of particles is always more than $10^{8}$, and it hinders the development of DEM. Now, some new methods are introduced into the research [18-23].

When the main structure and the damper are excited, the kinetic energy is dissipated through friction and inelastic collision between particles and damper. On this occasion, the powder mechanics is effective for calculating frictional energy dissipation, and the theory of collision energy is effective for calculating collisional energy dissipation for particle damper, which need less computation.

For the frictional motion, we established a model for particle damper based on powder mechanics, and the frictional energy dissipation was calculated. And for the inelastic collision motion, we established a collision model based on the motion of particles and the damper boundary. Then, the 


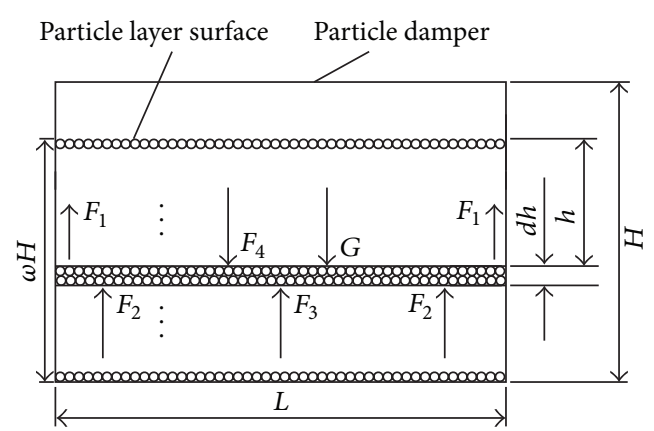

FIgURE 1: The forces on the particle layer $d h$.

total energy dissipation was calculated, and the simulation results were compared with experimental results. So the simulation model can provide a new analysis method for the practical application of particle damping technology.

\section{Model of Frictional Energy Dissipation}

The discrete element method can simulate the response of particle and damper with small numbers of particles. The large number of particles in the damper (in excess of $10^{8}$ ) will make the DEM method computationally very demanding. A simple assessment method considering layer's pressure is of important value in the design of damper with large number of particles.

In this paper, a parameter $K_{p}$ is introduced to describe the way, where the layer stress of the particles is redirected perpendicularly to its initial load [20].

If the total height of the rectangular particle damper is $H$, we establish a mechanical model. Figure 1 shows the particle layer with infinitesimal thickness $d h$. The forces on the particle layer $d h$ at the vertical direction are balanced by

$$
2 F_{1}+2 F_{2}+F_{3}-F_{4}-G=0
$$

where $F_{1}$ and $F_{2}$ represent the force of the damper side on the particle layer, $F_{3}$ represents the force on the lower particle layer, and $F_{4}$ represents the force on the upper particle layer.

The forces on the particle layer $d h$ in Figure 1 are given by

$$
\begin{aligned}
& F_{1}=\mu_{s} K_{p} \sigma_{p} L_{1} d h, \\
& F_{2}=\mu_{s} K_{p} \sigma_{p} L_{2} d h, \\
& F_{3}=\left(\sigma_{p}+d \sigma_{p}\right) L_{1} L_{2}, \\
& F_{4}=\sigma_{p} L_{1} L_{2}, \\
& G=\rho_{b} g L_{1} L_{2} d h,
\end{aligned}
$$

where $G$ represents the gravity of the particle layer, $\mu_{s}$ represents the friction coefficient between the inner damper and the particles, $K_{p}$ represents the Janssen coefficient, $\sigma_{p}$ represents the stress of the particle layer in the vertical direction, and $\rho_{b}$ represents the particle bulk density.

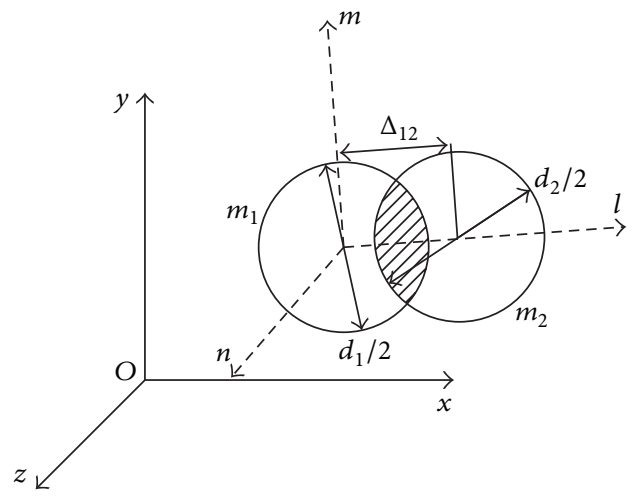

FIGURE 2: Coordinates of collision for adjacent particles.

Substituting (2) into (1), the integration can be rewritten as

$$
\sigma_{p}=\frac{\rho_{b} g L_{1} L_{2}}{2 \mu_{s} K_{p}\left(L_{1}+L_{2}\right)}\left[1-e^{-2 \mu_{s} K_{p}\left(L_{1}+L_{2}\right) h / L_{1} L_{2}}\right] .
$$

The distance of adjacent particles from the thinnest to the thickest is given by

$$
\Delta l=\left(\frac{4 \sqrt{3}}{3}-2\right) r .
$$

During the slippage of particles, on the basis of the energy conservation law, the kinetic energy for particle system will be dissipated due to friction work. As the particle system is under a harmonic excitation, the vibration of particle damper will be reduced.

Assuming the depth of particles is $h_{i}$ and the filling rate of particle volume is $\omega, h_{i}=\omega H$. It can be concluded that the maximum friction work $W_{f}$ for particle damper is given by

$$
\begin{array}{r}
W_{f}=\frac{2 \sqrt{3}-3}{6} \sum_{i=1}^{N} \frac{\mu_{p i} \rho_{b} g L_{1}^{2} L_{2}^{2} d}{\mu_{s} K_{p}\left(L_{1}+L_{2}\right)} \\
\cdot\left[1-e^{-2 \mu_{s} K_{p}\left(L_{1}+L_{2}\right) h_{i} / L_{1} L_{2}}\right],
\end{array}
$$

where $\mu_{p i}$ represents the friction coefficient of the particle layer $i, N$ represents the particle layer number in the damper, $N=\omega H / d, d$ represents the particle diameter, and $d=2 r$.

\section{Model of Collision Energy Dissipation}

In this paper, a model of collision energy dissipation for particle damper is established based on the collision theory.

3.1. The Model of Collision among the Particles. In order to judge the particle position at every moment, the local coordinates of interaction for the particles are established in Figure 2.

Assuming that the mass of particle 1 is $m_{1}$ and particle 2 is $m_{2}$ and the radius of particle 1 is $r_{1}$ and particle 2 is $r_{2}$, the position coordinates are, respectively, $\left(x_{1}, y_{1}, z_{1}\right)$ and 
$\left(x_{2}, y_{2}, z_{2}\right)$. So, the velocities are, respectively, $\left(x_{1}^{\prime}, y_{1}^{\prime}, z_{1}^{\prime}\right)$ and $\left(x_{2}^{\prime}, y_{2}^{\prime}, z_{2}^{\prime}\right)$. The contact condition for particles 1 and 2 is given by

$$
\begin{aligned}
d_{12} & =\sqrt{\left(x_{1}-x_{2}\right)^{2}+\left(y_{1}-y_{2}\right)^{2}+\left(z_{1}-z_{2}\right)^{2}} \\
& <\frac{d_{1}+d_{2}}{2} .
\end{aligned}
$$

Before the collision of particles, assuming that the velocity of particles 1 and 2 in the local coordinates is $\left(v_{1 l}, v_{1 m}, v_{1 n}\right)$ and $\left(v_{2 l}, v_{2 m}, v_{2 n}\right)$, the equations of the velocity for particle 1 between the original coordinates and local coordinates are given by

$$
\begin{aligned}
v_{1 l}= & x_{1}^{\prime} \cos \alpha+y_{1}^{\prime} \cos \beta+z_{1}^{\prime} \cos \gamma, \\
v_{1 m}= & -\frac{\cos \beta}{\sqrt{\cos ^{2} \alpha+\cos ^{2} \beta}} x_{1}^{\prime}-\frac{\cos \alpha}{\sqrt{\cos ^{2} \alpha+\cos ^{2} \beta}} y_{1}^{\prime}, \\
v_{1 n}= & -\frac{\cos \alpha \cdot \cos \gamma}{\sqrt{\cos ^{2} \alpha+\cos ^{2} \beta}} x_{1}^{\prime}-\frac{\cos \beta \cdot \cos \gamma}{\sqrt{\cos ^{2} \alpha+\cos ^{2} \beta}} y_{1}^{\prime} \\
& +z_{1}^{\prime} \sqrt{\cos ^{2} \alpha+\cos ^{2} \beta},
\end{aligned}
$$

where $\cos \alpha, \cos \beta$, and $\cos \gamma$ are the cosine of direction for $l$ axis.

After the collision of particles, based on the momentum theorem, the equation for particles 1 and 2 is given by

$$
\begin{aligned}
& m_{1} \cdot V_{1 m}+\lambda_{1} \cdot m_{2} \cdot V_{2 l}=m_{1} \cdot v_{1 m}+\lambda_{1} \cdot m_{2} \cdot v_{2 l}, \\
& m_{1} \cdot V_{1 n}+\lambda_{2} \cdot m_{2} \cdot V_{2 l}=m_{1} \cdot v_{1 n}+\lambda_{2} \cdot m_{2} \cdot v_{2 l},
\end{aligned}
$$

where $\left(V_{1 l}, V_{1 m}, V_{1 n}\right)$ and $\left(V_{2 l}, V_{2 m}, V_{2 n}\right)$ are the velocity components for particles 1 and 2 in the local coordinates, $\lambda_{i}$ is the friction coefficient in two directions, and $\lambda_{1}=I_{m} / I_{l}, \lambda_{2}=$ $I_{n} / I_{l}$.

$\lambda_{1}$ and $\lambda_{2}$ are given by $\operatorname{sgn} \lambda_{1}=\operatorname{sgn}\left(\left(v_{2 m}-v_{1 m}\right) /\left(v_{2 l}-v_{1 l}\right)\right)$ and $\operatorname{sgn} \lambda_{2}=\operatorname{sgn}\left(\left(v_{2 n}-v_{1 n}\right) /\left(v_{2 l}-v_{1 l}\right)\right)$.

The symbolic function sgn[·] is written as

$$
\operatorname{sgn}[x]= \begin{cases}1, & x \geq 0, \\ -1, & x \leq 0 .\end{cases}
$$

Assuming $V=\left\{V_{1 l}, V_{1 m}, V_{1 n}, V_{2 l}, V_{2 m}, V_{2 n}\right\}^{T}, v=\left\{v_{1 l}\right.$, $\left.v_{1 m}, v_{1 n}, v_{2 l}, v_{2 m}, v_{2 n}\right\}^{T}$.

The equation of velocity before and after collision of adjacent particles in local coordinates is given by

$$
V=[A]^{-1} \cdot[B] \cdot v,
$$

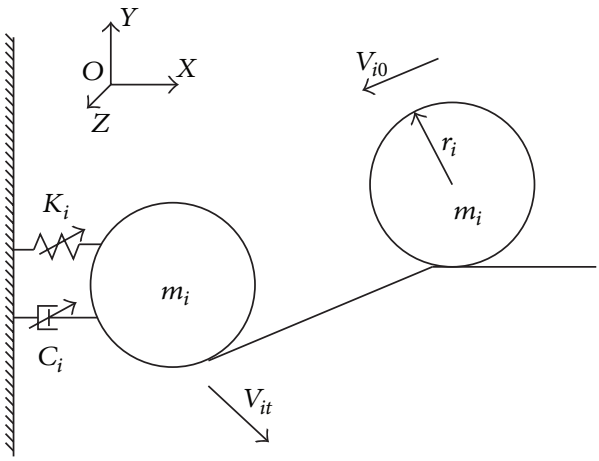

FIGURE 3: Model of collision between damper and particles.

where matrix $A$ and matrix $B$ are given by

$$
\begin{aligned}
{[A] } & =\left[\begin{array}{cccccc}
m_{1} & 0 & 0 & m_{2} & 0 & 0 \\
0 & m_{1} & 0 & 0 & m_{2} & 0 \\
0 & 0 & m_{1} & 0 & 0 & m_{2} \\
-1 & 0 & 0 & 1 & 0 & 0 \\
0 & m_{1} & 0 & -\lambda_{1} m_{1} & 0 & 0 \\
0 & 0 & m_{1} & -\lambda_{2} m_{2} & 0 & 0
\end{array}\right], \\
{[B] } & =\left[\begin{array}{cccccc}
m_{1} & 0 & 0 & m_{2} & 0 & 0 \\
0 & m_{1} & 0 & 0 & m_{2} & 0 \\
0 & 0 & m_{1} & 0 & 0 & m_{2} \\
-e & 0 & 0 & e & 0 & 0 \\
0 & m_{1} & 0 & -\lambda_{1} m_{1} & 0 & 0 \\
0 & 0 & m_{1} & -\lambda_{2} m_{2} & 0 & 0
\end{array}\right] .
\end{aligned}
$$

When two particles collide, one particle will move to a new position. After a time step $\Delta t$, the new coordinates of position for particle 1 are given by

$$
\begin{aligned}
& x_{1 t}=x_{1}+\frac{\left(x_{1 t}^{\prime}+x_{1}^{\prime}\right)}{2} \Delta t, \\
& y_{1 t}=y_{1}+\frac{\left(y_{1 t}^{\prime}+y_{1}^{\prime}\right)}{2} \Delta t, \\
& z_{1 t}=z_{1}+\frac{\left(z_{1 t}^{\prime}+z_{1}^{\prime}\right)}{2} \Delta t .
\end{aligned}
$$

3.2. The Model of Collision between the Damper and Particles. As the particles keep moving in the damper, the particles collide with the inner damper. The contact between the damper and the particles can be done as the mass-springdamping system.

Assume that the mass for particle $i$ is $m_{i}$, and its motion velocity is $v_{i}$. The collision process can be divided into two phases "compression and recovery phase" [14]. Assuming that the motion velocity of particle $i$ is $v_{i t}$ after the collision, the model of collision is given in Figure 3.

3.2.1. Compression Phase. During the compression phase, based on the theorem of impulse, the equation of impulse is written as 


$$
\begin{aligned}
m x_{i 0}^{\prime c}-m x_{i 0}^{\prime} & =I_{c x}, \\
m y_{i 0}^{\prime c}-m y_{i 0}^{\prime} & =I_{c y}, \\
0-m z_{i 0}^{\prime} & =I_{c z},
\end{aligned}
$$

where $\left(x_{i 0}^{\prime}, y_{i 0}^{\prime}, z_{i 0}^{\prime}\right)$ is the motion velocity of particle $m_{i}$ in the tangent and the normal plane before the compression; $\left(x_{i 0}^{\prime c}, y_{i 0}^{\prime c}, z_{i 0}^{\prime c}\right)$ is the motion velocity of particle $m_{i}$ in the tangent and the normal plane after the compression; $\left(I_{c x}, I_{c y}, I_{c z}\right)$ is the impulse of particle $m_{i}$ in the tangent and the normal plane during the compression phase.

After the compression phase, the component $v_{i 0 \tau}^{c}$ of motion velocity for particle $m_{i}$ in tangent plane is

$$
v_{i 0 \tau}^{c}=\sqrt{\left(x_{i 0}^{\prime}\right)^{2}+\left(y_{i 0}^{\prime}\right)^{2}}-\mu_{c} z_{i 0}^{\prime},
$$

where the dynamic friction coefficient $\mu_{c}$ in the tangent plane is defined by

$$
\mu_{c}=\sqrt{\frac{I_{c x}^{2}+I_{c y}^{2}}{I_{c z}^{2}}} .
$$

3.2.2. Recovery Phase. During the recovery phase, the equation of collision impulse is written as

$$
\begin{aligned}
m x_{i t}^{\prime}-m x_{i 0}^{\prime c} & =I_{r x}, \\
m y_{i t}^{\prime}-m y_{i 0}^{\prime c} & =I_{r y}, \\
m z_{i t}^{\prime}-0 & =I_{r z},
\end{aligned}
$$

where $\left(x_{i t}, y_{i t}, z_{i t}\right)$ is the position of particle $m_{i}$ in the tangent and the normal plane after the recovery; $\left(x_{i t}^{\prime}, y_{i t}^{\prime}, z_{i t}^{\prime}\right)$ is the motion velocity of particle $m_{i}$ in the tangent and the normal plane after the recovery; $\left(I_{r x}, I_{r y}, I_{r z}\right)$ is the impulse of particle $m_{i}$ in the tangent and the normal plane during the recovery phase.

The Newton recovery coefficient $e_{0}$ in the normal plane at the contact area between particle $m_{i}$ and the damper is defined by

$$
e_{0}=\frac{V_{2 l}-V_{1 l}}{v_{1 l}-v_{2 l}} .
$$

After the recovery phase, the motion velocity $v_{i t \tau}$ for particle $m_{i}$ in the tangent plane becomes

$$
v_{i t \tau}=\sqrt{\left(x_{i}^{\prime}\right)^{2}+\left(y_{i}^{\prime}\right)^{2}}-\mu_{c}\left(1+e_{0}\right) z_{i}^{\prime} .
$$

After a whole collision process $\Delta t$, the new coordinates of position for particle $m_{i}$ are given by

$$
\begin{aligned}
& x_{i t}=x_{i 0}+\frac{\left(x_{i 0}^{\prime}+x_{i t}^{\prime}\right)}{2} \cdot \Delta t, \\
& y_{i t}=y_{i 0}+\frac{\left(y_{i 0}^{\prime}+y_{i t}^{\prime}\right)}{2} \cdot \Delta t, \\
& z_{i t}=z_{i 0}+r_{i} .
\end{aligned}
$$

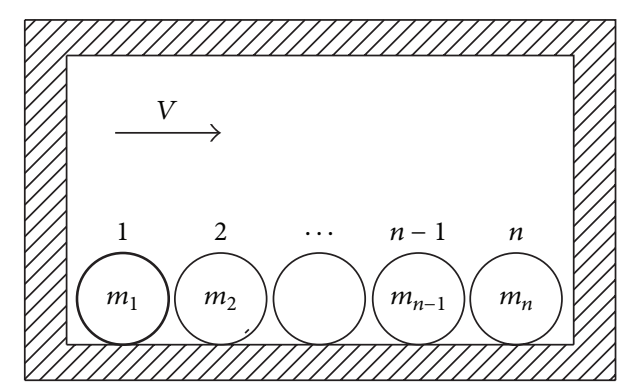

FIGURE 4: The model after collision with the inner damper.

3.3. The Model after Collision with the Inner Damper. After the collision with the inner damper for particle $m_{1}$, Figure 4 shows the model of the adjacent particles.

Assuming that the motion velocity of particle 1 is $v_{1}$, particle 2 is static during the collision between particle 1 and the inner damper. After the collision with particle 2, based on the momentum theorem, the motion velocity $v_{2}$ of particle 2 is given by

$$
v_{2}=\frac{m_{1}\left(1+e_{12}\right) v_{1}}{m_{1}+m_{2}},
$$

where $e_{12}$ is the recovery coefficient between particle 1 and particle 2.

In Figure 4, the energy of particle 1 is transferred and attenuated from particle 1 to particle $n$. By the recursion, the motion velocity $v_{n}$ during the collision of the $n$th particle is given by

$$
v_{n}=\prod_{i=2}^{n} \frac{m_{i-1}\left[1+e_{(i-1) i}\right]}{m_{i}+m_{i-1}} \cdot v_{1} .
$$

Before and after the collision, assume that the motion velocity for the damper is $V_{1}$ and $V_{2}$. The recovery coefficient $e_{0}$ can be calculated by (17), and the motion velocity $v_{n}$ is written as

$$
v_{n}=\prod_{i=2}^{n} \frac{m_{i-1}\left[1+e_{(i-1) i}\right]}{m_{i}+m_{i-1}} \cdot\left[e_{0} V_{1}+V_{2}\right] .
$$

From (22), we can see that the velocity $v_{n}$ is associated with the particle number, the particle material, the damper material, and the damper velocity before the collision.

As the motion of particle changes, it will access the next step size of time and return to the above process, which calculates the increment of motion for particle again and again. By the iterative calculation, we can achieve the real time motion tracing for every particle.

For the particle damper, the energy dissipation of collision motion is defined as

$$
W_{c}=\sum_{n=1}^{N_{t}}\left[\frac{1}{2} m_{n} v_{n 0}^{2}-\frac{1}{2} m_{n} v_{n t}^{2}\right],
$$

where $N_{t}$ represents the particle number participating collision. 


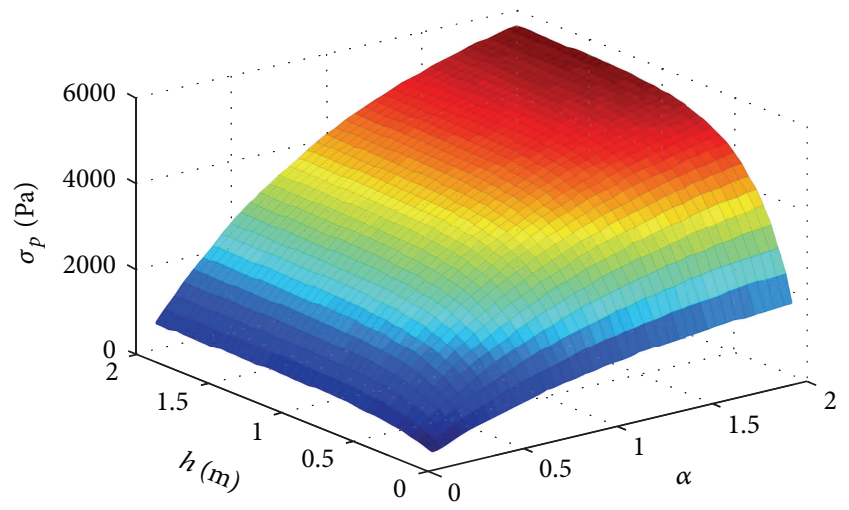

FIGURE 5: The variation of $\sigma_{p}$ with $\alpha$ and $h$.

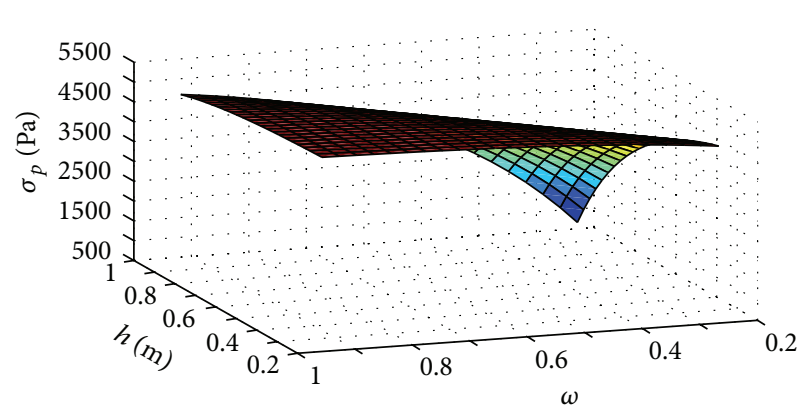

FIGURE 6: Variation of $\sigma_{p}$ with $h$ and $\omega$.

For the damper model, the total energy dissipation $W_{T}$ of particles is given by

$$
W_{T}=W_{f}+W_{c}
$$

\section{Result Analysis and Experimental Verification}

4.1. Discussion of Parameters for Friction Model. Assuming that $\mu_{s}=0.45$ (magnesium oxide to steel), $K_{p}=0.7, \mu_{p i}=$ 0.25 (magnesium oxide), and $\rho=1.65 \mathrm{~g} / \mathrm{cm}^{3}$. Figure 5 shows the variation of the stress $\sigma_{p}$ with the depth $h$ of the particle layer and the ratio $\alpha$ of the length $L_{1}$ to the width $L_{2}(\alpha=$ $L_{1} / L_{2}$ ) for the particle damper. As the depth $h$ increases, the stress of the particle layer intensifies. When the layer depth $h$ exceeds a value, the motion amplitude of the whole particle keeps steady. As the particle depth $h$ is $1.35 \mathrm{~m}$, the limiting value of the stress $\sigma_{p}$ is $4752 \mathrm{~Pa}$. This particle layer can bear the weight of the entire particle system. And, as shown in Figure 5, at the same depth of particle layer, with the increase of the ratio $\alpha$ for the damper, the stress $\sigma_{p}$ increases.

Figure 6 shows the effects of layer depth $h$ and filling rate $\omega$ on the stress $\sigma_{p}$. With the increase of filling rate $\omega$, the stress $\sigma_{p}$ at the same depth of particle layer increases. But with the increase of filling rate $\omega$, the total particle number by frictional motion in the damper below reduces, weakening the damping effect.

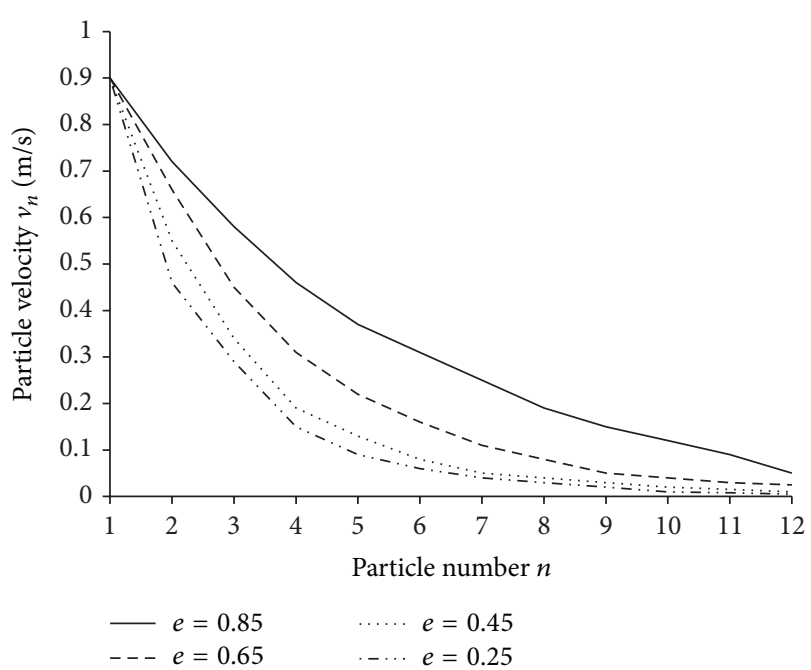

FIGURE 7: Effects of particle number $n$ and recovery coefficients $e$ on velocity $v_{n}$.

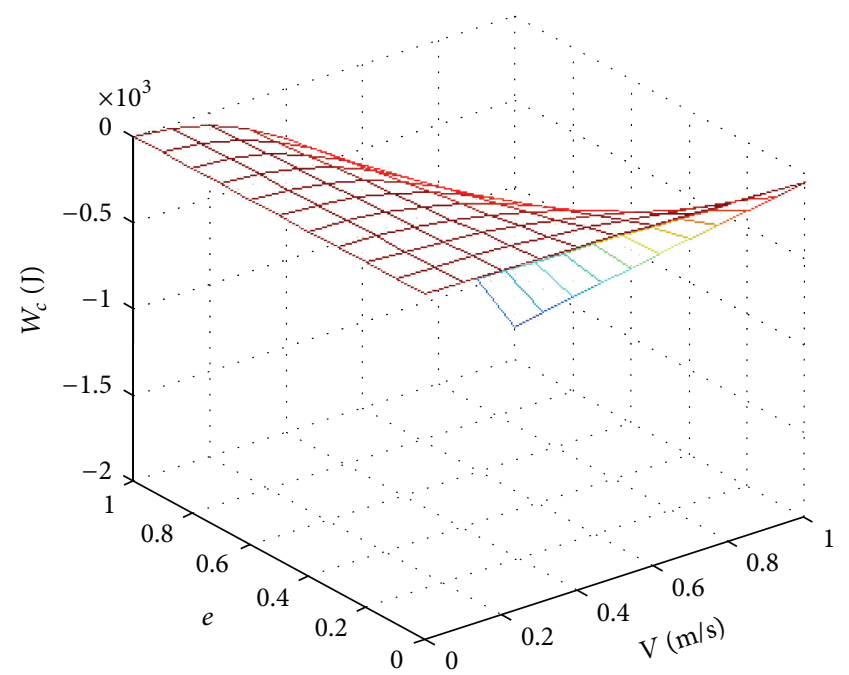

Figure 8: Effects of recovery coefficients $e$ and initial velocity $V$ on energy dissipation $W_{c}$.

4.2. Discussion of Parameters for Collision Model. Figure 7 shows the effects of the particle number $n$ and the recovery coefficients $e$ on the velocity of adjacent particles. The recovery coefficients of particles are $0.25,0.45,0.65$, and 0.85. As shown in Figure 7, after collision between particles and damper, the velocity for the adjacent particle drops quickly. A lower recovery coefficient will lead to a greater energy dissipation and damping effect. When the collision is transferred to the seventh particle and $e$ is less than 0.65 , the particle velocity will drop to $6 \%$ initial velocity.

Figure 8 shows the effects of the recovery coefficients $e$ and the initial velocity $V$ on the energy dissipation $W_{c}$. When the initial velocity for particle damper increases, the energy dissipation rises. A higher initial velocity will result in a faster energy attenuation. As the recovery coefficient increases, the damping effect weakens in the particle damper. 


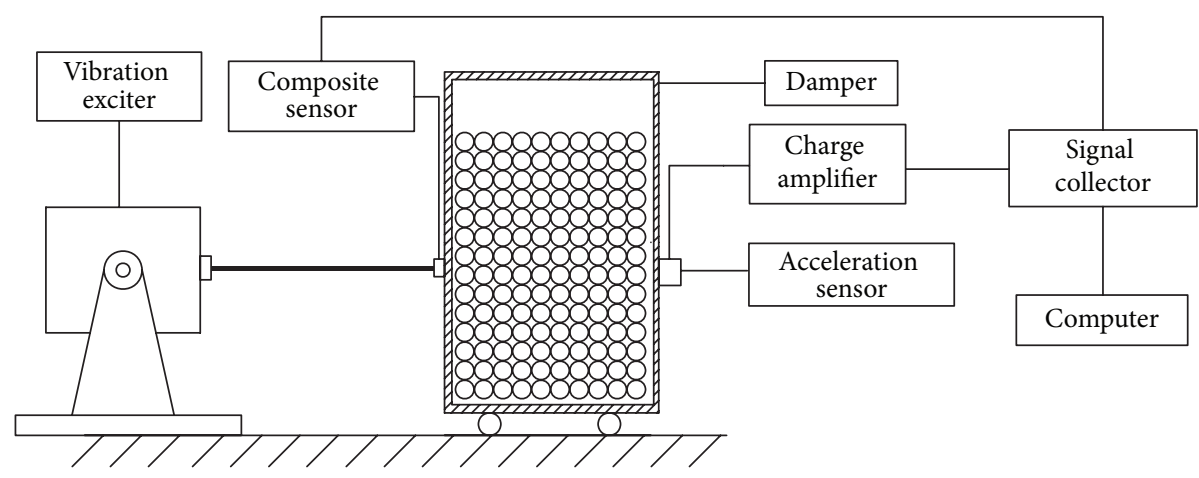

FIGURE 9: The schematic plot of experimental system.

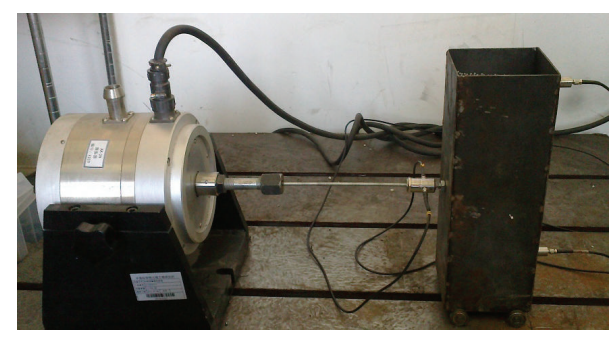

FIGURE 10: The photograph of experimental system.

4.3. Experimental Verification. The energy dissipation experiment is performed to verify the correctness of the model for particle damping. The shape of experimental damper is a cube, and the damper material is carbon steel, with the dimensions of $150 \mathrm{~mm} \times 150 \mathrm{~mm} \times 400 \mathrm{~mm}$. The schematic plot of experimental system is shown in Figure 9.

The test setup is mainly composed of test components, electromagnetic exciter of JZ-30, acceleration sensors, and signal acquisition analyzer of INV-3118. The electromagnetic exciter produces the excitation signal through two-force bar. The acceleration signal of the damper is acquired by a signal collection and transferred to a signal analyzer. The sampling number per cycle is more than 500 and the experimental process is repeated 4 times. The excitation signal of the electromagnetic vibrator JZ-30 is the sine wave.

In this experiment, the energy dissipation for particles is written as

$$
W_{\text {exp }}=W_{\text {exc }}-W_{\text {kin }}
$$

where $W_{\exp }$ represents the energy dissipation for particles in this experiment, $W_{\text {exc }}$ represents the excitation work of damper which could be calculated by the force gauge, and $W_{\text {kin }}$ represents the kinetic energy which could be calculated by the acceleration sensor.

The photograph of this experimental system is shown in Figure 10 .

Firstly, the aluminum oxide, the stainless steel, and the tungsten carbide are used for experimental material. The densities of the three materials are, respectively, $3.4 \mathrm{~g} / \mathrm{cm}^{3}$, $7.9 \mathrm{~g} / \mathrm{cm}^{3}$, and $18.4 \mathrm{~g} / \mathrm{cm}^{3}$ with $85 \%$ volume filling rate and

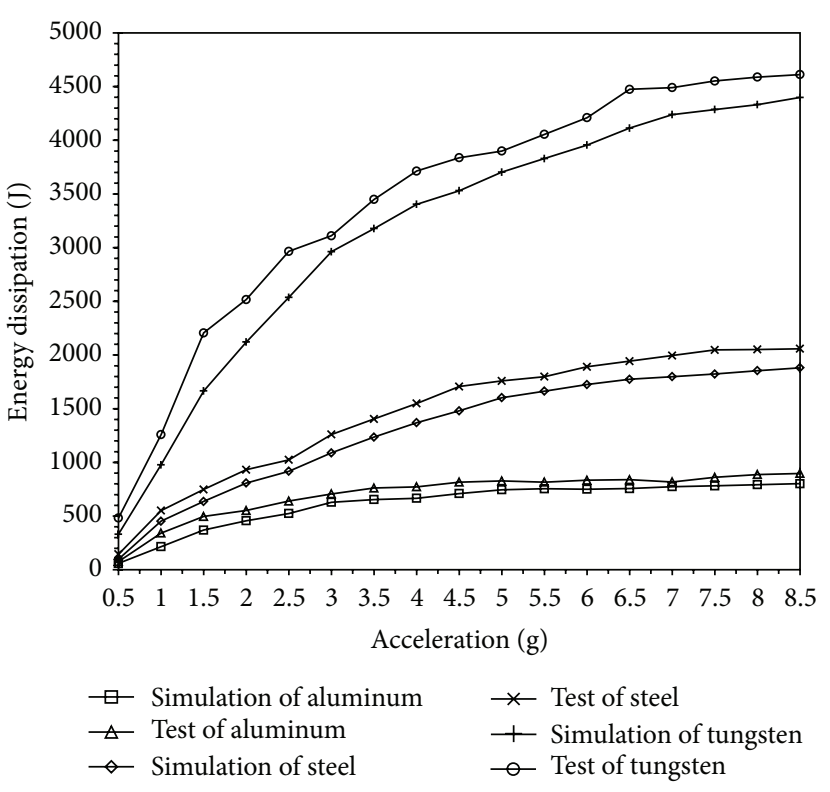

FIGURE 11: The energy dissipation results for the simulation and experiment.

$4 \mathrm{~mm}$ particle size. The results showing the energy dissipation versus acceleration distributions for the simulation and experiment are shown in Figure 11 under the three densities of particle. The distributions for the energy dissipation of particles versus acceleration are nonlinear. For the low density of particle, the energy dissipation is less than the high density of particle. As the particle density increases, the damping effect rises, and the vibration attenuation of the tungsten carbide particle material is optimal in the experiment.

From Figure 11, the distributions of the energy dissipation for the simulation and test are in good agreement, and the experimental values of energy dissipation are slightly more than the simulation results. It verifies the rationality for the theoretical model of particle damper. As the experiment process includes lots of factors of energy dissipation, such as the noise and the air resistance, the experimental value is about $7 \%$ more than the simulation value. So, for the design process of particle damper, the simulation model can provide an effective method to choose an appropriate particle density. 


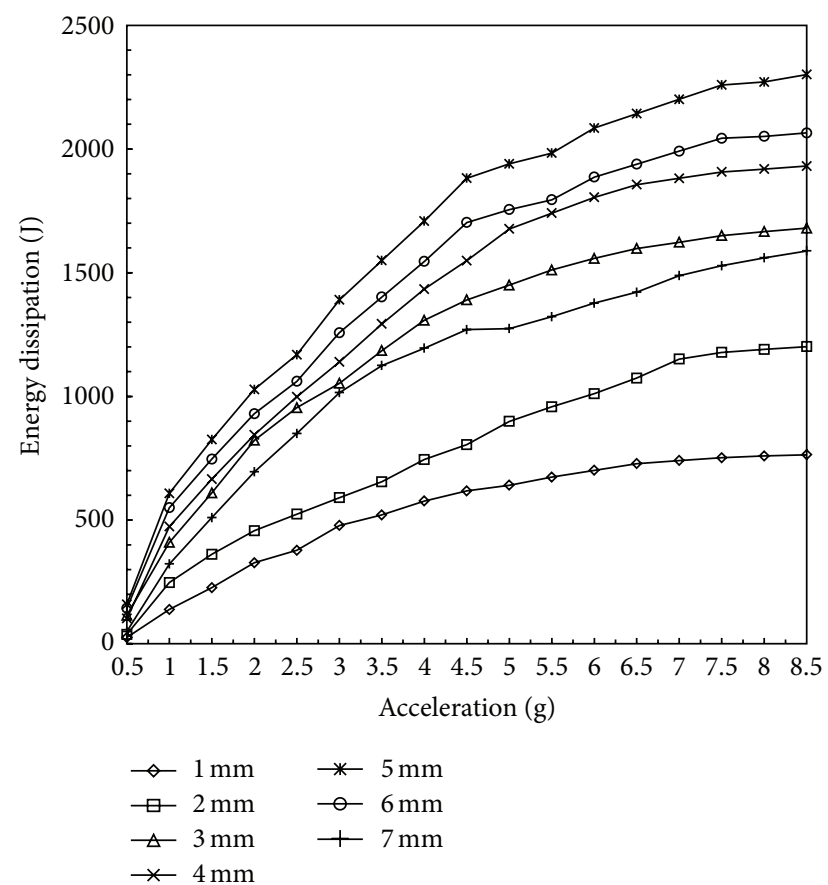

FIgURE 12: The experimental results for the different particle size.

Secondly, in order to investigate the effect of particle size and exciting acceleration on the energy dissipation, the stainless steel is used for experimental material with $85 \%$ volume filling rate. The experimental results showing the energy dissipation versus acceleration distributions for the different particle size are shown in Figure 12. As the particle size increases from $1 \mathrm{~mm}$ to $5 \mathrm{~mm}$, the energy dissipation rises greatly. And as the particle size increases from $5 \mathrm{~mm}$ to $7 \mathrm{~mm}$, the energy dissipation drops. So we can draw the conclusion that there is an optimum particle size for vibration attenuation of a structure. In this experiment, the $5 \mathrm{~mm}$ particle size is the best for stainless steel ball. One reason for this result is that there is the adhesion among the too small particle size; and another reason is that too large particle size will result in fewer chances for friction and collision, which will decrease the vibration attenuation of an exciting structure. So, for the design process of particle damper, choosing an appropriate particle size determined based on the simulation model is important.

Thirdly, in order to investigate the effect of particle filling rate and exciting acceleration on the energy dissipation, the stainless steel is used for experimental material $4 \mathrm{~mm}$ particle size. The experimental results showing the energy dissipation versus acceleration distributions for the different particle filling rate are shown in Figure 13.

As there is no particle in the damper, the energy dissipation value is nearly zero. And as the particle filling rate increases to $90 \%$, the energy dissipation values rise. However, as the particle filling rate exceeds $90 \%$, the energy dissipation values begin to drop. So we can see that there is an optimum particle filling rate for damping effect. In this experiment, the $90 \%$ particle filling rate is the best for stainless steel ball of

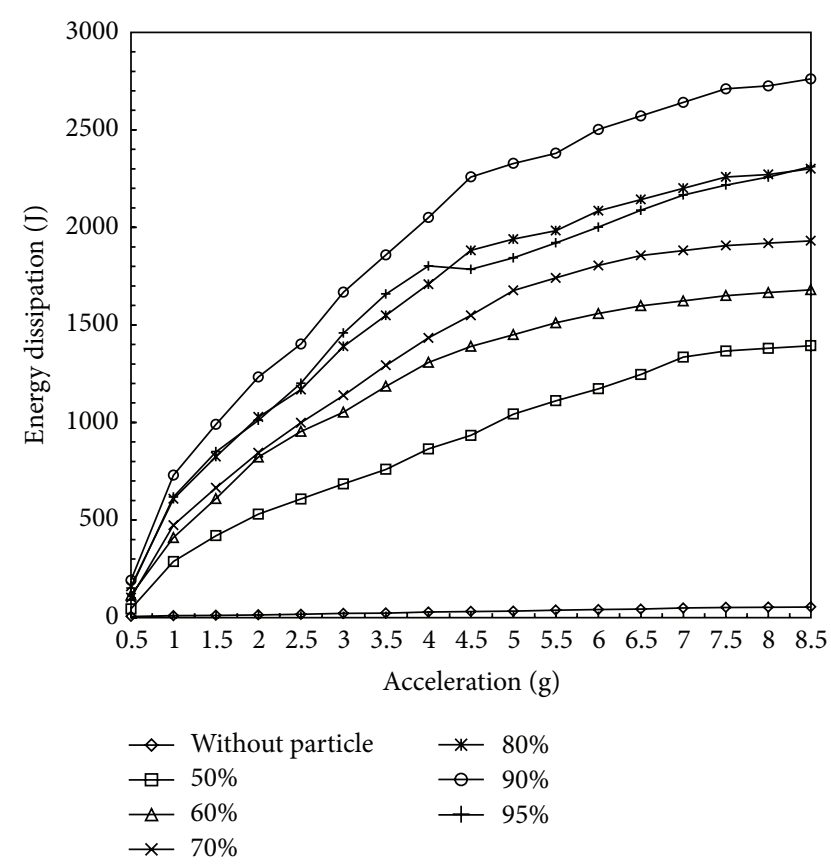

FIGURE 13: The experimental results for the different particle filling rate.

$4 \mathrm{~mm}$ particle size. The main reason for this result is that there is less clearance of particle motion in the damper for too much particle, which will decrease the damping effect of an exciting structure. So, choosing an appropriate particle filling rate based on the simulation model will provide for the practical application of particle damping technology.

\section{Conclusions}

An energy dissipation model for particle damper has been established based on the powder mechanics and the collision theory. The equations of friction energy dissipation and collision energy dissipation are developed for the particle damper. Through the experiment, the rationality of energy dissipation model is verified and the important parameters of particle damping have been investigated, such as the particle density, the particle size, and the particle filling rate.

(1) The distributions for the energy dissipation of particles versus acceleration are nonlinear. For the low density of particle, the energy dissipation is less than the high density of particle. As the particle density increases, the damping effect rises, and the vibration attenuation of the tungsten carbide particle material is optimal in the experiment.

(2) As the particle size increases from $1 \mathrm{~mm}$ to $5 \mathrm{~mm}$, the energy dissipation rises greatly, and as the particle size increases from $5 \mathrm{~mm}$ to $7 \mathrm{~mm}$, the energy dissipation drops. In this experiment, the $5 \mathrm{~mm}$ particle size is the best for stainless steel ball.

(3) As the particle filling rate increases to $90 \%$, the energy dissipation values rise. However, as the particle filling rate exceeds $90 \%$, the energy dissipation values begin to drop. In 
this experiment, the $90 \%$ particle filling rate is the best for stainless steel ball of $4 \mathrm{~mm}$ particle size.

For the design process of particle damper, choosing an appropriate particle density, particle size, and particle filling rate determined based on the simulation model will provide the optimal damping effect for the practical application of particle damping technology.

\section{Conflict of Interests}

The authors declare that there is no conflict of interests regarding the publication of this paper.

\section{Acknowledgments}

The authors would like to acknowledge financial support from National Natural Science Foundation of China (no. 51205382) and the Fundamental Research Funds for the Central Universities, Xiamen University (no. 20720150094), and Collaborative Innovation Center of High-End Equipment Manufacturing in Fujian.

\section{References}

[1] X.-M. Bai, B. Shah, L. M. Keer, Q. J. Wang, and R. Q. Snurr, "Particle dynamics simulations of a piston-based particle damper," Powder Technology, vol. 189, no. 1, pp. 115-125, 2009.

[2] K. Li and A. P. Darby, "Experiments on the effect of an impact damper on a multiple-degree-of- freedom system," Journal of Vibration and Control, vol. 12, no. 5, pp. 445-464, 2006.

[3] Z. Lu, X. Lu, and S. F. Masri, "Studies of the performance of particle dampers under dynamic loads," Journal of Sound and Vibration, vol. 329, no. 26, pp. 5415-5433, 2010.

[4] M. R. Duncan, C. R. Wassgren, and C. M. Krousgrill, "The damping performance of a single particle impact damper," Journal of Sound and Vibration, vol. 286, no. 1-2, pp. 123-144, 2005.

[5] B. M. Shah, D. Pillet, X. M. Bai, L. M. Keer, Q. Jane Wang, and R. Q. Snurr, "Construction and characterization of a particlebased thrust damping system," Journal of Sound and Vibration, vol. 326, no. 3-5, pp. 489-502, 2009.

[6] H. V. Panossian, "Non-obstructive particle damping tests on aluminum beams," in Proceedings of the Damping 91 Conference, pp. 13-17, San Diego, Calif, USA, 1991.

[7] D. Guyomar and A. Badel, "Nonlinear semi-passive multimodal vibration damping: an efficient probabilistic approach," Journal of Sound and Vibration, vol. 294, no. 1-2, pp. 249-268, 2006.

[8] A. R. Jem and R. T. Geoffrey, "Amplitude dependent behaviour in the application of particle dampers to vibrating structures," Proceedings of the 46th AIAA/ASME/ASCE/AHS/ASC Structures, Structural Dynamics, and Materials Conference, pp. 18-21, 2005.

[9] C. H. Lamarque and O. Janin, "Modal analysis of mechanical systems with impact non-linearities: limitations to a modal superposition," Journal of Sound and Vibration, vol. 235, no. 4, pp. 567-609, 2000.

[10] B. K. Mishra and C. V. R. Murty, "On the determination of contact parameters for realistic DEM simulations of ball mills," Powder Technology, vol. 115, no. 3, pp. 290-297, 2001.

[11] J. Chen, L.-B. Wang, and X.-M. Huang, "Micromechanical modeling of asphalt concrete fracture using a user-defined three-dimensional discrete element method," Journal of Central South University, vol. 19, no. 12, pp. 3595-3602, 2012.

[12] S. T. Nase, W. L. Vargas, A. A. Abatan, and J. J. McCarthy, "Discrete characterization tools for cohesive granular material," Powder Technology, vol. 116, no. 2-3, pp. 214-223, 2001.

[13] Z.-Y. Xie and J.-X. Feng, "Numerical analysis of behavior of active layer in rotary kilns by discrete element method," Journal of Central South University, vol. 20, no. 3, pp. 634-639, 2013.

[14] B. M. Shah, J. J. Nudell, K. R. Kao, L. M. Keer, Q. J. Wang, and K. Zhou, "Semi-active particle-based damping systems controlled by magnetic fields," Journal of Sound and Vibration, vol. 330, no. 2, pp. 182-193, 2011.

[15] K. S. Marhadi, Particle impact damping: influence of material and size [Master's thesis], Texas A\&M University, 2003.

[16] Y. Y. Michael, Development of master design curves for particle impact damper [Ph.D. thesis], Pennsylvania State University, 2003.

[17] A. R. Jem and G. R. Tomlinson, "Amplitude dependent behaviour in the application of particle dampers to vibrating structures," in Proceedings of the 46th AIAA/ ASME/ASCE/AHS/ASC Structures, Structural Dynamics \& Materials Conference, vol. 10, pp. 6433-6441, Austin, Tex, USA, April 2005.

[18] C. Shi, S. N. Wang, L. Liu, Q. X. Meng, and Q. Zhang, "Mesomechanical simulation of direct shear test on outwash deposits with granular discrete element method," Journal of Central South University, vol. 20, no. 4, pp. 1094-1102, 2013.

[19] D. I. Ryzhkov, "Vibration damper for metal cutting," The Engineer's Digest, vol. 14, pp. 246-251, 2003.

[20] W. Liu, G. R. Tomlinson, and J. A. Rongong, "The dynamic characterisation of disk geometry particle dampers," Journal of Sound and Vibration, vol. 280, no. 3-5, pp. 849-861, 2005.

[21] L. Wu and F.-Z. Qu, "Discrete element simulation of mechanical characteristic of conditioned sands in earth pressure balance shield tunneling," Journal of Central South University of Technology, vol. 16, no. 6, pp. 1028-1033, 2009.

[22] G. B. Gong and X. X. Zha, "DEM simulation of liquefaction for cohesionless media at grain scale," Journal of Central South University, vol. 19, no. 9, pp. 2643-2649, 2012.

[23] K. Mao, M. Y. Wang, Z. Xu, and T. Chen, "DEM simulation of particle damping," Powder Technology, vol. 142, no. 2-3, pp. 154$165,2004$. 

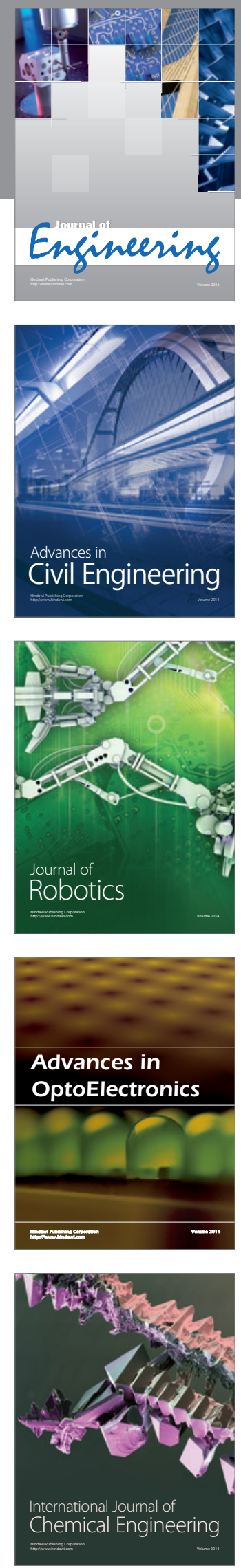

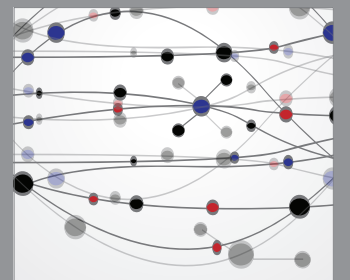

The Scientific World Journal
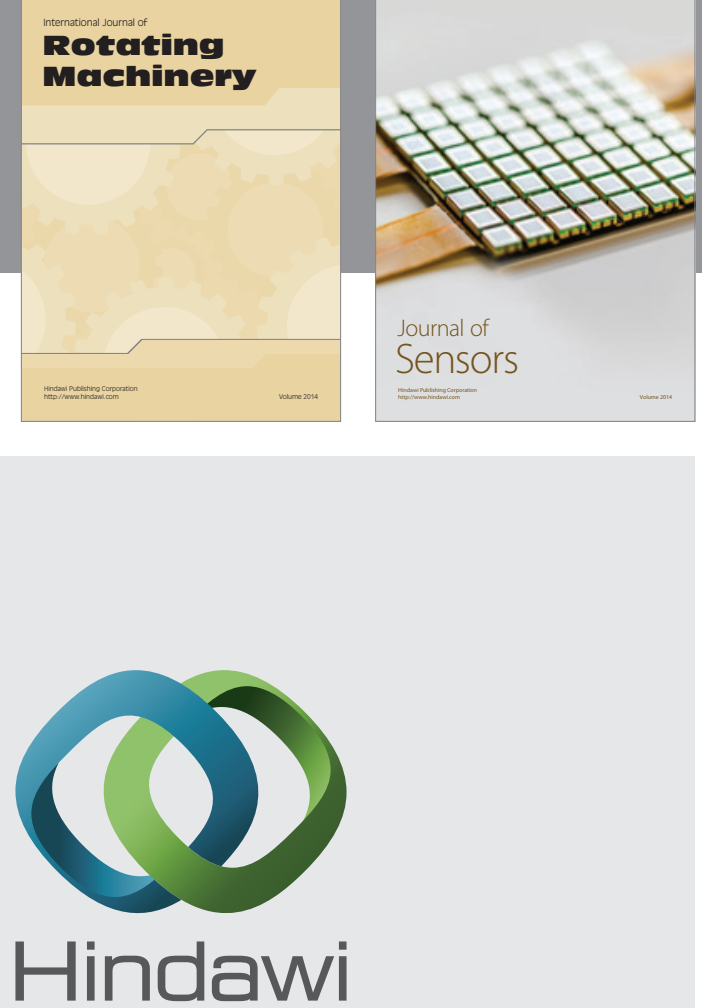

Submit your manuscripts at http://www.hindawi.com
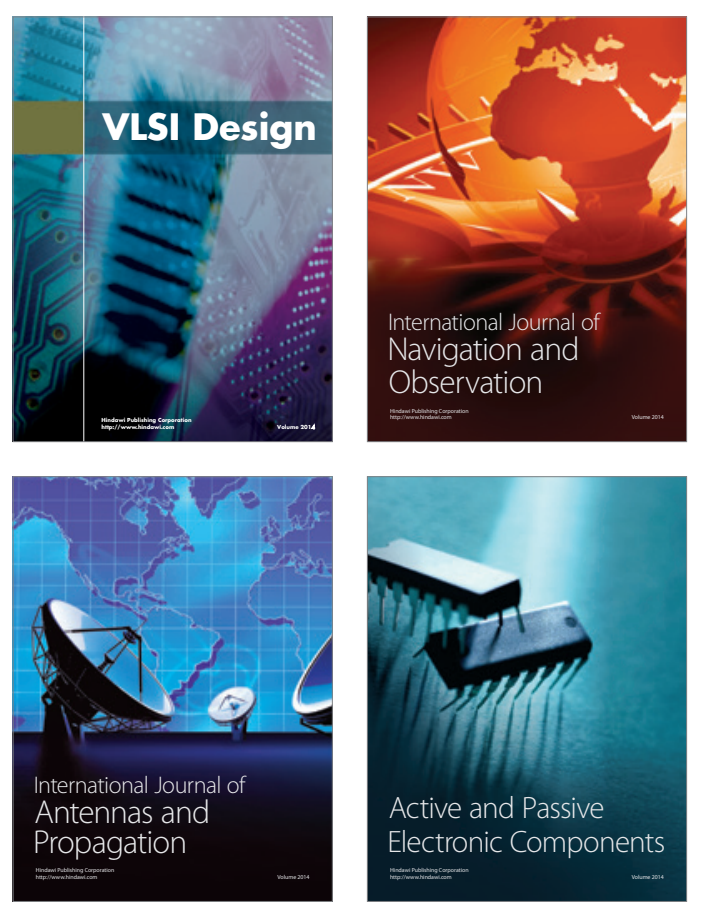
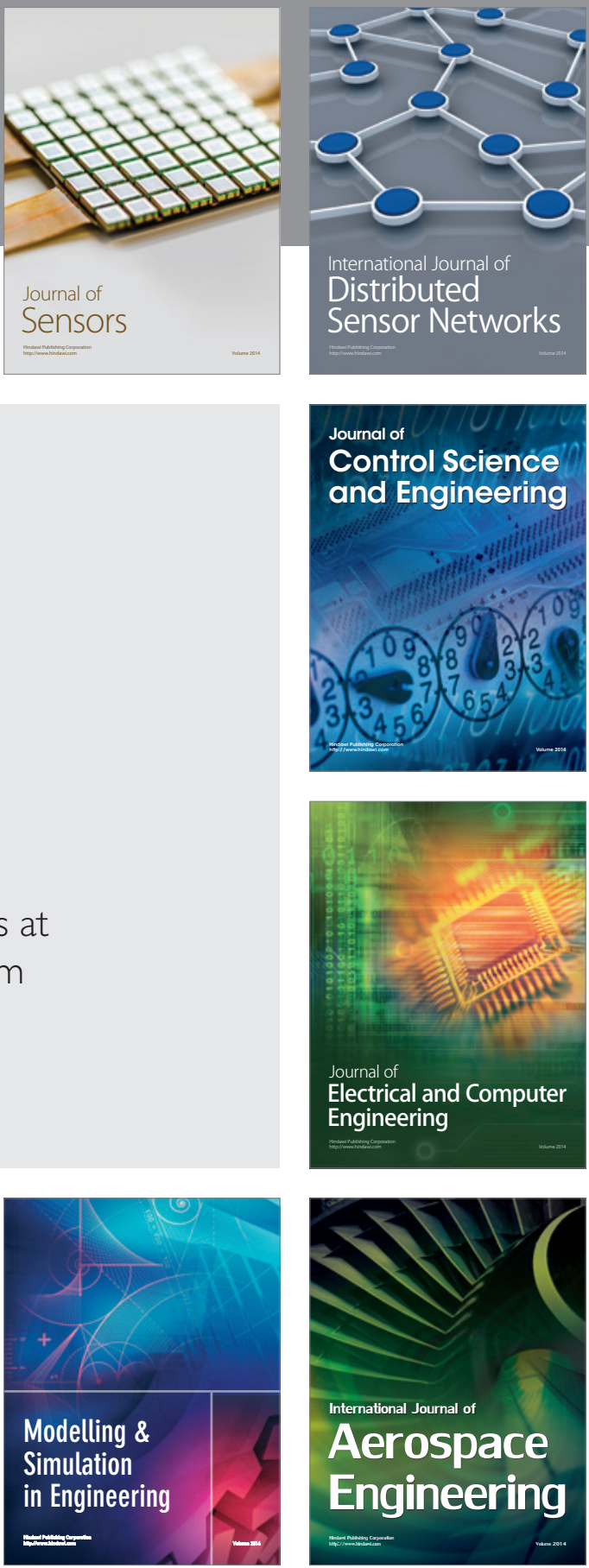

Journal of

Control Science

and Engineering
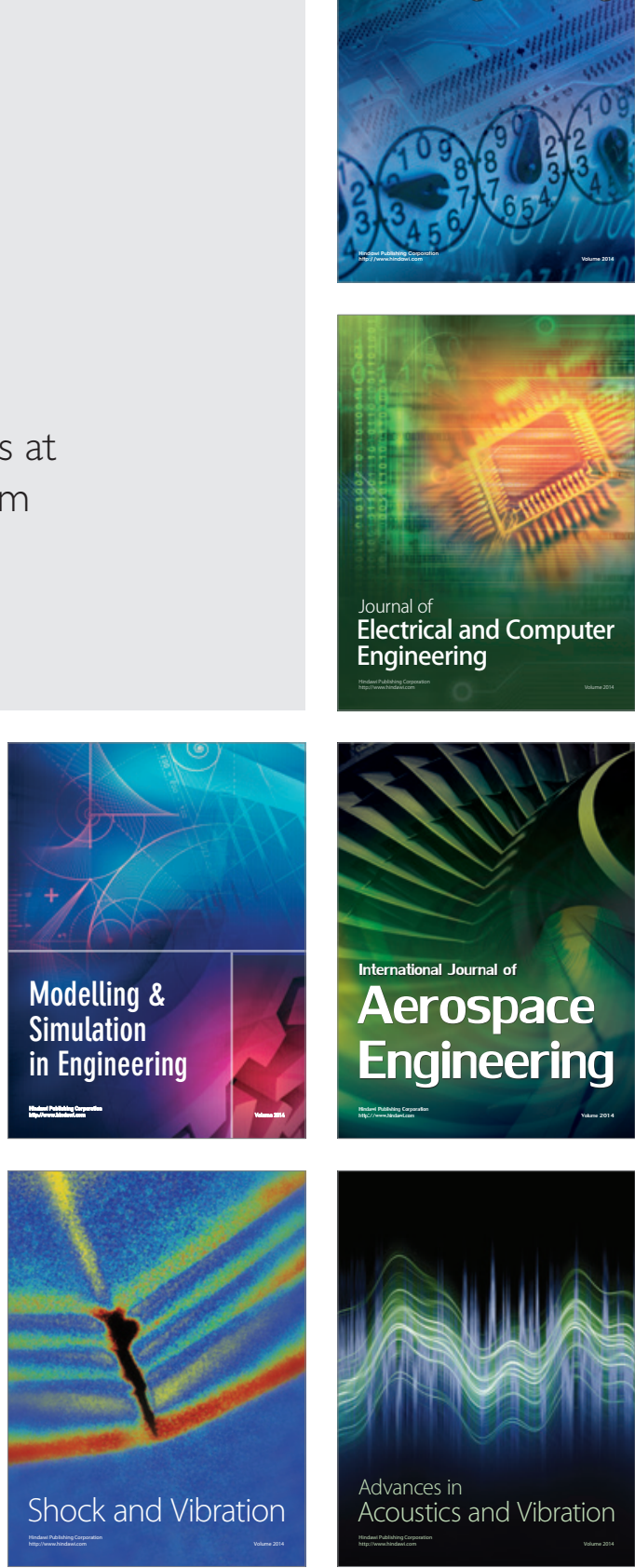